Pacific Journal of Mathematics

DISCONJUGACY OF LINEAR DIFFERENTIAL EQUATIONS IN 


\title{
DISCONJUGACY OF LINEAR DIFFERENTIAL EQUATIONS IN THE COMPLEX DOMAIN
}

\author{
Meira Lavie
}

\begin{abstract}
Necessary conditions for disconjugacy of $n$-th order linear differential equations in the unit disk, as well as sufficient conditions for $m-m$ disconjugacy of self-adjoint equations are obtained. Invariants of the differential equation under Möbius transformations are used and some examples are considered.
\end{abstract}

Let $p_{2}(z), \cdots, p_{n}(z)$ be regular functions in the simply-connected domain $D$, which does not contain $z=\infty$. The differential equation

$$
y^{(n)}(z)+\left(\begin{array}{l}
n \\
2
\end{array}\right) p_{2}(z) y^{(n-2)}(z)+\cdots+p_{n}(z) y(z)=0
$$

is called disconjugate in $D$, if no (nontrivial) solution of (1.1) has $n$ zeros in $D$. (The zeros are counted by their multiplicity.) Equation (1.1) is said to be $m-m$ disconjugate in $D$ if $n=2 m$ and no (nontrivial) solution of (1.1) has two zeros of order $m$ in $D$.

In $\S 2$ we consider the effect of a linear Möbius transformation of the independent variable $z$ on the form of equation (1.1). Modifying a result of Wilczynski [10], we assert (Theorem 1) the existence of certain combinations of the coefficients of equation (1.1) which remain invariant under the group of linear Möbius transformations. These invariants, which we denote by $I_{j}(z), j=2, \cdots, n$, play an important role in our study of disconjugacy properties of equation (1.1).

Making use of Theorem 1, we obtain in $\S 3$ bounds for all the coefficients of the disconjugate equation (1.1) and all its invariants. Thus, we prove (Theorem 2) that if equation (1.1) is disconjugate in $|z|<1$, then

$$
\left|I_{j}(z)\right| \leqq \frac{A(j, n)}{\left(1-|z|^{2}\right)^{j}}, \quad|z|<1, j=2, \cdots, n
$$

and

$$
\left|p_{j}(z)\right| \leqq \frac{B(j, n)}{\left(1-|z|^{2}\right)^{j}}, \quad|z|<1, j=2, \cdots, n,
$$

where $A(j, n)$ and $B(j, n)$ are constants which depend only on $j$ and $n$. Theorem 2 extends a former result [7, Th. 5], where a bound was given only for the first nonvanishing coefficient of the disconjugate equation (1.1). 
By a procedure essentially due to Fano [3], we obtain in $\S 4$ a differential equation of the type (1.1), such that this $n$-th order equation and the second order equation

$$
w^{\prime \prime}(z)+s(z) w(z)=0
$$

are simultaneously disconjugate or not disconjugate in $D$. Using then a result of Hille [4], we show that (1.2) and (1.3) are of the correct order of growth.

Finally, in $\S 5$, we generalize a recent result of Kim [6, Th. 2.1], and give (Theorem 3) sufficient conditions for $m-m$ disconjugacy of self-adjoint differential equations of order $2 \mathrm{~m}$. This is done by utilizing again the existence of the invariants (Theorem 1) as well as a sharp integral inequality obtained by $\operatorname{Kim}[6, \mathrm{Th} .3 .3]$.

2. Linear invariants associated with equation (1.1). We start with a remark concerning the form of equation (1.1) and the choice of the domain $D$. Consider the differential equation

$$
\begin{aligned}
& y^{(n)}(z)+\left(\begin{array}{c}
n \\
1
\end{array}\right) p_{1}(z) y^{(n-1)}(z)+\left(\begin{array}{c}
n \\
2
\end{array}\right) p_{2}(z) y^{(n-2)}(z) \\
& +\cdots+p_{n}(z) y(z)=0,
\end{aligned}
$$

where $p_{j}(z), j=1,2, \cdots, n$, are regular functions in the simply-connected domain $D$, not containing $z=\infty$. Let $\zeta(z)$ be a regular one-to-one analytic transformation which maps the domain $D$ onto the domain 4. Set

$$
y(z)=w[\zeta(z)] \tau(z), \quad \tau(z) \neq 0 .
$$

It is easily verified that by making a proper choice of $\tau(z)$, say

$$
\tau(z)=\left[\zeta^{\prime}(z)\right]^{(1-n) / 2} \exp \left[\int^{z}-p_{1}(t) d t\right],
$$

equation (2.1) is transformed into the differential equation

$$
w^{(n)}(\zeta)+\left(\begin{array}{l}
n \\
2
\end{array}\right) q_{2}(\zeta) w^{(n-2)}(\zeta)+\cdots+q_{n}(\zeta) w(\zeta)=0
$$

Furthermore, (2.3) is disconjugate in $\Delta$, if and only if (2.1) is disconjugate in $D$. Hence, without loss of generality, we may assume, as we did in (1.1), that the coefficient of $y^{(n-1)}(z)$ is identically zero. Moreover, it is sufficient to consider disconjugacy properties of equation (1.1) in the unit disk. This will be done in §'s 3 and 5 .

Suppose now that $\zeta(z)$ is regular and one to one in $D$, and set

$$
y(z)=w[\zeta(z)]\left[\zeta^{\prime}(z)\right]^{(1-n) / 2} .
$$


Equation (1.1) is transformed by the substitution (2.4) into equation (2.3), and we are concerned now with the relations between the coefficients of these equations.

For second order differential equations $(n=2)$ it is well known (e.g. see [5, p. 394]) that

$$
p_{2}(z)=q_{2}[\zeta(z)]\left[\zeta^{\prime}(z)\right]^{2}+\frac{1}{2}\{\zeta(z), z\},
$$

where

$$
\{\zeta(z), z\}=\frac{\zeta^{\prime \prime \prime}(z)}{\zeta^{\prime}(z)}-\frac{3}{2}\left[\frac{\zeta^{\prime \prime}(z)}{\zeta^{\prime}(z)}\right]^{2}
$$

is the Schwarzian derivative. For higher order differential equations $(n>2)$, a similar relation holds [10, p. 24]; namely,

$$
p_{2}(z)=q_{2}[\zeta(z)]\left[\zeta^{\prime}(z)\right]^{2}+\frac{(n+1)}{6}\{\zeta(z), z\} .
$$

$\left(2.5^{\prime}\right)$ can be verified directly; see also [7, Ths. 3 and 4]. As is well known, the Schwarzian derivative (2.6) vanishes identically, if and only if $\zeta(z)$ is a linear transformation of the form

$$
\zeta(z)=\frac{a z+b}{c z+d}, \quad a d-b c \neq 0 .
$$

In this case, $\left(2.5^{\prime}\right)$ is reduced to

$$
p_{2}(z)=q_{2}[\zeta(z)]\left[\zeta^{\prime}(z)\right]^{2} .
$$

We say now, that $p_{2}(z)$ is an "invariant of weight 2 " of the differential equation (1.1) under linear transformations of the type (2.7).

Simple relations like (2.8) do not hold between the other coefficients of equations (1.1) and (2.3). However, (2.8) turns out to be the simplest case of the following theorem.

THEOREM 1. Let equation (1.1) be transformed into equation (2.3) by the substitution (2.4), where $\zeta(z)$ is given by (2.7). Then, for every index $j, 2 \leqq j \leqq n$, there exists a linear combination

$$
\begin{aligned}
I_{j}(z) & =L_{j}\left[p_{2}(z), \cdots, p_{j}(z)\right] \\
& =\sum_{s=2}^{j} a_{j, s} p_{s}^{(j-s)}(z), j=2, \cdots, n,
\end{aligned}
$$

such that

$$
I_{j}(z)=J_{j}[\zeta(z)]\left[\zeta^{\prime}(z)\right]^{j}, \quad 2 \leqq j \leqq n,
$$

where 


$$
J_{j}(\zeta)=L_{j}\left[q_{2}(\zeta), \cdots, q_{j}(\zeta)\right]=\sum_{s=2}^{j} a_{j, s} q_{s}^{(j-s)}(\zeta)
$$

The coefficients $a_{j, s}$ are given by

$$
\begin{aligned}
a_{j, s} & =\frac{(-1)^{j-s} j !(j-1) !(j+s-2) !}{s !(s-1) !(j-s) !(2 j-2) !}, \\
s & =2, \cdots, j, j=2, \cdots, n,
\end{aligned}
$$

and are uniquely determined up to a multiplicative constant.

Thus, Theorem 1 asserts the existence of invariants of weights $2,3, \cdots, n$ of equation (1.1) when subject to a transformation (2.7).

Invariants associated with linear differential equations were studied by Brioschi, Forsyth, Fano, Wilczynski and others. In [2], Brioschi considered general transformations $\zeta(z)$ and established the existence of nonlinear invariants of weights $3,4, \cdots, 7$. These invariants may be reduced to linear invariants of the form (2.9), if $\zeta(z)$ is assumed to be a linear transformation of the form (2.7). As we have already seen, (2.8) also holds only for $\zeta(z)$ of the type (2.7). Wilczynski [10, p. 26-32] considers linear transformations $\zeta(z)$, but he assumes that $p_{2}(z) \equiv 0$. However, by applying slight modifications to Wilczynski's proof, one can show that it actually works even if $p_{2}(z) \not \equiv 0$, and thus establish Theorem 1.

REMARK. We note that the coefficients $p_{j}(z), j=2, \cdots, n$, of equation (1.1) not only determine the invariants $I_{j}(z), j=2, \cdots, n$, but are also uniquely determined by them. Indeed, if $I_{j}(z), j=2, \cdots, n$, are given regular functions in the domain $D$, it follows from the very form of (2.9) that

$$
p_{2}(z)=I_{2}(z), p_{3}(z)=I_{3}(z)-a_{3,2} p_{2}^{\prime}(z)=I_{3}(z)+\frac{3}{2} I_{2}^{\prime}(z) .
$$

Thus, successive elimination of $p_{2}(z), \cdots, p_{j}(z)$ from $(2.9)$ leads us to

$$
p_{j}(z)=\sum_{s=2}^{j} b_{j, s} I_{s}^{(j-s)}(z), j=2, \cdots, n
$$

where the constants $b_{j, s}, s=2, \cdots, j, j=2, \cdots, n$, are uniquely determined by (2.11). More specifically, if we complete the schemes of constants $a_{j, s}$ and $b_{j, s}, s=2, \cdots, j, j=2, \cdots, n$, given by (2.11) and (2.12) respectively, by setting $a_{j, s}=0, b_{j, s}=0$ for $s=j+1, \cdots, n$, $j=2, \cdots, n$ we obtain two triangular matrices $A=\left[a_{j, s}\right]_{2}^{n}$ and $B=$ $\left[b_{j, 3}\right]_{2}^{n}$, and $B$ is the inverse of $A$.

We add the following corollaries to Theorem 1 . 
CoRollary 1. Let equation (1.1) be transformed to equation (2.3) by the substitution (2.4), where $\zeta(z)$ is given by (2.7). If the coefficients of equation (1.1) are such that

$$
p_{2}(z) \equiv p_{3}(z) \equiv \cdots \equiv p_{k-1}(z) \equiv 0, p_{k}(z) \not \equiv 0,2 \leqq k \leqq n,
$$

then the coefficients of equation (2.3) satisfy a similar relation; namely

$$
q_{2}(\zeta) \equiv q_{3}(\zeta) \equiv \cdots \equiv q_{k-1}(\zeta) \equiv 0, \quad 2 \leqq k \leqq n
$$

and

$$
p_{k}(z)=q_{k}[\zeta(z)]\left[\zeta^{\prime}(z)\right]^{k} \text {. }
$$

(cf. [10, p. 26]), [7, Th. 4], [6, Corollary 2.1].).

COROLlaRY 2. Let

$$
\left.y^{(n)}(z)+\left(\begin{array}{l}
n \\
2
\end{array}\right) p_{2}^{*}(z) y^{(n-2)}\right)(z)+\cdots+p_{n}^{*}(z) y(z)=0,
$$

be the adjoint equation of (1.1), and let $I_{j}^{*}(z)$ and $I_{j}(z), j=2, \cdots, n$, be the invariants of equations (2.13) and (1.1) respectively. Then

$$
I_{j}^{*}(z)=(-1)^{j} I_{j}(z), \quad j=2, \cdots, n .
$$

By the definition of the adjoint equation, (2.13) is given by

$$
\begin{aligned}
y^{(n)}(z) & +\left(\begin{array}{l}
n \\
2
\end{array}\right)\left[p_{2}(z) y(z)\right]^{(n-2)} \\
& -\left(\begin{array}{l}
n \\
3
\end{array}\right)\left[p_{3}(z) y(z)\right]^{(n-3)}+\cdots+(-1)^{n} p_{n}(z) y(z)=0 .
\end{aligned}
$$

Hence

$$
p_{2}^{*}(z)=p_{2}(z), p_{3}^{*}(z)=-p_{3}(z)+3 p_{2}^{\prime}(z),
$$

and in general

$$
p_{t}^{*}(z)=(-1)^{t} p_{t}(z)+\sum_{r=2}^{t-1} c_{t, r} p_{r}^{(t-r)}(z), t=2, \cdots, n .
$$

Expressing $p_{t}^{*}(z)$ in terms of $p_{r}^{(t-r)}(z), r=2, \cdots, t-1$, by means of (2.15) and substituting in $I_{j}^{*}(t)$, we obtain a linear combination of

$$
p_{s}^{(j-s)}(z), s=2, \cdots, j
$$

which is an invariant of weight $j$. Since by Theorem 1 the linear invariant of weight $j$ is uniquely determined up to a constant factor, 
it follows that $I_{j}^{*}(z)=k_{j} I_{j}(z), j=2, \cdots, n$. The constants $k_{j}, j=$ $2, \cdots, n$, are determined by the coefficient of $p_{j}(z)$ in $I_{j}^{*}(z)$; hence $k_{j}=$ $(-1)^{j}$. (cf. [2, p. 237], [10, p. 46].)

COROLlaRY 3. In order that equation (1.1) will be self-adjoint, it is necessary and sufficient that all the invariants of odd weight vanish identically; i.e.,

$$
I_{2 i+1}(z) \equiv 0, i=1,2, \cdots\left[\frac{n-1}{2}\right] .
$$

If equation (1.1) is self-adjoint then (2.16) follows from (2.14). Conversely, if (2.16) holds then the differential equation is selfadjoint. Indeed, by (2.16) and (2.14) the invariants of the given equation coincide with the respective invariants of the adjoint equation. Since the coefficients $p_{j}(z)$ are uniquely determined by the invariants, (see the remark following the proof of Theorem 1) it follows that the differential equation coincides with its adjoint.

COROLlaRY 4. If $\zeta(z)$ is given by (2.7), then the substitution (2.4) transforms adjoint equations into adjoint equations. In particular, equation (2.3) is self-adjoint if and only if equation (1.1) is.

Theorem 1 and its corollaries play an important role in our study of disconjugacy of equation (1.1) in the unit disk. We note that the most general one-to-one analytic transformation which maps $|z|<1$ onto $|\zeta|<1$ is given by

$$
\zeta(z)=\frac{e^{i \theta}\left(z-z_{0}\right)}{1-z \bar{z}_{0}},\left|z_{0}\right|<1,0 \leqq \theta<2 \pi,|z|<1 .
$$

For every choice of the parameters $z_{0}$ and $\theta$ in (2.17), equation (1.1) is transformed by the substitution (2.4) into a differential equation of the type (2.3). Since disconjugacy is preserved by this transformation, both equations are either disconjugate or not disconjugate in the unit disk. Finally since (2.17) is of the type (2.7), Theorem 1 can be applied to yield the relations between the coefficients of equations (1.1) and (2.3). Furthermore, any necessary condition for disconjugacy should be satisfied not only by the coefficients of equation (1.1) but by the coefficients of equation (2.3) as well. Hence, as will become apparent in the following sections, it seems more intrinsic to express disconjugacy conditions in terms of the invariants $I_{j}(z)$ rather than in terms of the coefficients $p_{j}(z)$.

3. Necessary conditions for disconjugacy. We apply now The- 
orem 1 in order to obtain necessary conditions for disconjugacy of equation (1.1) in the unit disk.

THEOREM 2. Let the coefficients $p_{j}(z), j=2, \cdots, n$ of equation (1.1) be regular in $|z|<1$, and assume that (1.1) is disconjugate in $|z|<1$. Then, there exist constants $A(j, n)$ and $B(j, n)$, depending only on $j$ and $n$, such that

$$
\begin{aligned}
\left|I_{j}(z)\right| & =\left|\sum_{s=2}^{j} a_{j, s} p_{s}^{(j-s)}(z)\right| \\
& \leqq \frac{A(j, n)}{\left(1-|z|^{2}\right)^{j}},|z|<1, j=2, \cdots, n,
\end{aligned}
$$

and

$$
\left|p_{j}(z)\right| \leqq \frac{B(j, n)}{\left(1-|z|^{2}\right)^{j}},|z|<1, j=2, \cdots, n .
$$

In particular

$$
A(2, n)=B(2, n)=(n+1),
$$

and this result is sharp. Moreover, for $j=3, \cdots, n,(3.1)$ and (3.2) are of the correct order.

We remark that the necessary conditions for disfocality of equation (1.1) in $|z|<1$, obtained in [8, Th. 7], are of the same order as (3.2).

The following lemma will be required in the proof of Theorem 2 .

LEMma 1. Let $h_{k}(z), k=1,2, \cdots$, be a regular function in $|z|<$ 1. If

$$
\left|h_{k}(z)\right| \leqq \frac{1}{\left(1-|z|^{2}\right)^{k}},|z|<1
$$

then

$$
\left|h_{k}^{(s)}(z)\right| \leqq \frac{C(s, k)}{\left(1-|z|^{2}\right)^{s+k}},|z|<1, s=1,2, \cdots,
$$

where $C(s, k)$ are constants depending only on $s$ and $k$.

Lemma 1 can be proved by applying the Cauchy integral formula for the derivatives. While in general we shall be concerned only with the existence of the constants $C(s, k)$ and not with their magnitude, it is worth noting that better estimates for the constants $C(s, k)$ are obtained by a method given in [8, Lemma 4]. 
Proof of Theorem 2. Let

$$
y_{1}(z)=z^{n-2}\left[1+\sum_{t=2}^{\infty} \alpha_{t} z^{t}\right],|z|<1,
$$

and

$$
y_{2}(z)=z^{n-1}\left[1+\sum_{t=2}^{\infty} \beta_{t} z^{t}\right],|z|<1,
$$

be two solutions of equation (1.1). Substituting (3.6) and (3.7) in equation (1.1), the constants $\alpha_{t}$ and $\beta_{t}, t=2,3, \cdots$, are determined by the coefficients $p_{j}(z), j=2, \cdots, t$, of (1.1) in the following way:

$$
\begin{aligned}
\alpha_{2}= & -\frac{p_{2}(0)}{2}, \beta_{2}=-\frac{(n-1)}{2(n+1)} p_{2}(0), \\
\alpha_{3}= & -\frac{(n-2) p_{3}(0)+3 p_{2}^{\prime}(0)}{3 !(n+1)}, \\
\beta_{3}= & -\frac{(n-1)(n-2) p_{3}(0)+6(n-1) p_{2}^{\prime}(0)}{3 !(n+1)(n+2)}, \\
\alpha_{4}= & -\frac{(n-2)(n-3) p_{4}(0)+8(n-2) p_{3}^{\prime}(0)+12 p_{2}^{\prime \prime}(0)-6 n(n-1) p_{2}^{2}(0)}{4 !(n+1)(n+2)}, \\
\beta_{4}= & -\frac{(n-1)(n-2)(n-3) p_{4}(0)+12(n-1)(n-2) p_{3}^{\prime}(0)}{4 !(n+1)(n+2)(n+3)} \\
& -\frac{36(n-1) p_{2}^{\prime \prime}(0)-6 n(n-1)^{2} p_{2}^{2}(0)}{4 !(n+1)(n+2)(n+3)},
\end{aligned}
$$

and

$$
\begin{aligned}
& \alpha_{t}=-\frac{n !(n-2) ! p_{t}(0)}{t !(n-t) !(n+t-2) !}+\left.Q_{t}\left[p_{j}^{(s)}(z)\right]\right|_{z=0}, t=3, \cdots, n \\
& \beta_{t}=-\frac{n !(n-1) ! p_{t}(0)}{t !(n-t) !(n+t-1) !}+\left.\widetilde{Q}_{t}\left[p_{j}^{(s)}(z)\right]\right|_{z=0}, t=3, \cdots, n
\end{aligned}
$$

where $Q_{t}$ and $\widetilde{Q}_{t}$ are polynomials of the arguments

$$
p_{j}^{(s)}(z), s=0, \cdots t-j, j=2, \cdots, t-1 .
$$

Since equation (1.1) is disconjugate in $|z|<1$, it follows from [7, Th. 1] that the function

$$
f(z)=\frac{y_{1}(z)}{y_{2}(z)}=z^{-1}\left[1+\sum_{t=2}^{\infty} \gamma_{t} z^{t}\right],|z|<1,
$$

is univalent in $|z|<1$. This assertion can easily be confirmed. Indeed, suppose that $f\left(z_{1}\right)=f\left(z_{2}\right)=a b^{-1}$, where $\left|z_{1}\right|,\left|z_{2}\right|<1$, then the non- 
trivial solution $a y_{1}(z)-b y_{2}(z)$ has $(n-2)$ zeros at the origin (this follows readily from (3.6) and (3.7)) and two zeros at $z_{1}$ and $z_{2}$. But this contradicts our assumption that equation (1.1) is disconjugate in $|z|<1$.

According to (3.9) the coefficients $\gamma_{t}$ are given by

$$
\gamma_{2}=\alpha_{2}-\beta_{2}, \gamma_{3}=\alpha_{3}-\beta_{3}, \gamma_{4}=\alpha_{4}-\beta_{4}+\beta_{2}^{2}-\alpha_{2} \beta_{2},
$$

and

$\left(3.10^{\prime}\right) \quad \gamma_{t}=\alpha_{t}-\beta_{t}+\Gamma_{t}\left[\alpha_{2}, \cdots, \alpha_{t-1}, \beta_{2}, \cdots, \beta_{t-1}\right], t=4,5, \cdots$,

where $\Gamma_{t}$ is a polynomial of the specified arguments. Insertion of (3.8) and $\left(3.8^{\prime}\right)$ in $(3.10)$ and $\left(3.10^{\prime}\right)$ leads us to

$$
\begin{aligned}
\gamma_{2}=- & \frac{p_{2}(0)}{n+1}, \gamma_{3}=-\frac{(n-2)}{2(n+1)(n+2)} \\
& \times\left[p_{3}(0)-\frac{n-4}{n-2} p_{2}^{\prime}(0)\right], \\
\gamma_{t}= & -\frac{n !(n-2) ! p_{t}(0)}{(n-t) !(t-1) !(n+t-1) !} \\
& +\left.G_{t}\left[p_{j}^{(s)}(z)\right]\right|_{z=0}, t=3,4, \cdots, n,
\end{aligned}
$$

where $G_{t}$ is a polynomial of the arguments $p_{j}^{(s)}(z), j=2, \cdots, t-1$, $s=0, \cdots, t-j$.

Having established the relations between the coefficients $\gamma_{t}$ of the function $f(z)$ and the coefficients $p_{j}(z)$ of the differential equation (1.1), we are ready to proceed with our proof. As has already been mentioned, disconjugacy of equation (1.1) in the unit disk implies the univalence of the function (3.9) there. Applying now the area-theorem to the coefficients of the univalent function (3.9), we obtain

$$
\sum_{t=2}^{\infty}(t-1)\left|\gamma_{t}\right|^{2} \leqq 1
$$

Hence,

$$
\left|\gamma_{t}\right| \leqq(t-1)^{-1 / 2}, t=2,3, \cdots .
$$

Combining (3.11) and (3.13) we shall obtain upper bounds for

$$
\left|p_{2}(0)\right|, \cdots,\left|p_{n}(0)\right| \text {. }
$$

Utilizing then Theorem 1 and Lemma 1, (3.1) and (3.2) will be established by an induction on $j$. We proceed now with the details.

Setting $t=2$ in (3.13), it follows by (3.11) that

$$
\left|p_{2}(0)\right| \leqq(n+1) \text {. }
$$


Applying now the transformation (2.17), equation (1.1) is transformed by the substitution (2.4) into equation (2.3). According to Theorem 1 and (2.17) we now have

$$
I_{j}\left(z_{0}\right)=J_{j}(0)\left[\zeta^{\prime}\left(z_{0}\right)\right]^{j}, j=2, \cdots, n,
$$

where $I_{j}(z)$ and $J_{j}(z)$ are the invariants of equations (1.1) and (2.3) respectively. For $j=2$, it follows from (3.15) that

$$
p_{2}\left(z_{0}\right)=I_{2}\left(z_{0}\right)=J_{2}(0)\left[\zeta^{\prime}\left(z_{0}\right)\right]^{2}=q_{2}(0)\left[\zeta^{\prime}\left(z_{0}\right)\right]^{2} .
$$

Since disconjugacy is preserved by a transformation of the type (2.17), equation (2.3) is disconjugate in $|\zeta|<1$. Hence, according to (3.14)

$$
\left|q_{2}(0)\right| \leqq(n+1) \text {. }
$$

In view of the fact that for transformations of the type (2.17)

$$
\left|\zeta^{\prime}(z)\right|=\frac{1-|\zeta|^{2}}{1-|z|^{2}},|z|<1,
$$

it follows from $\left(3.14^{\prime}\right)$ and (3.16) that

$$
\left|I_{2}\left(z_{0}\right)\right|=\left|p_{2}\left(z_{0}\right)\right| \leqq \frac{(n+1)}{\left(1-\left|z_{0}\right|^{2}\right)^{2}},\left|z_{0}\right|<1 .
$$

Since (3.18) holds for every $\left|z_{0}\right|<1$, this completes the proof for $j=2$.

Next, we consider $j=3$. For $t=3$, (3.11) and (3.13) yield

$$
\left|p_{3}(0)-\frac{n-4}{n-2} p_{2}^{\prime}(0)\right| \leqq \frac{\sqrt{2}(n+1)(n+2)}{(n-2)} .
$$

By the Cauchy inequality, it follows from (3.18) that

$$
\left|p_{2}^{\prime}(0)\right| \leqq(n+1) \operatorname{Min}_{0 \leqq r<1}\left\{r^{-1}\left(1-r^{2}\right)^{-2}\right\}=\frac{(n+1) 25 \sqrt{5}}{16} .
$$

Combining (3.19) and (3.20), we obtain

$$
\begin{aligned}
\left|p_{3}(0)\right| & \leqq \frac{(n+1)}{(n-2)}\left[\sqrt{2}(n+2)+\frac{25 \sqrt{5}}{16}(n-4)\right] \\
& =B_{0}(3, n)
\end{aligned}
$$

and

$$
\begin{aligned}
\left|I_{3}(0)\right| & =\left|p_{3}(0)-\frac{3}{2} p_{2}^{\prime}(0)\right| \\
& \leqq\left(\frac{25 \sqrt{5}}{32}+\sqrt{2}\right) \frac{(n+1)(n+2)}{(n-2)}=A(3, n) .
\end{aligned}
$$


Since by our assumptions equation (2.3) is disconjugate, it follows from (3.22) that

$$
\left|J_{3}(0)\right| \leqq A(3, n) \text {. }
$$

Combining now (3.15), (3.17) and $\left(3.22^{\prime}\right)$, we obtain that

$\left(3.22^{\prime \prime}\right)$

$$
\begin{aligned}
\left|I_{3}\left(z_{0}\right)\right| & =\left|p_{3}\left(z_{0}\right)-\frac{3}{2} p_{2}^{\prime}\left(z_{0}\right)\right| \\
& \left.=\left|J_{3}(0)\right|\left|\zeta^{\prime}\right| z_{0}\right)\left.\right|^{3} \leqq \frac{A(3, n)}{\left(1-\left|z_{0}\right|^{2}\right)^{3}},\left|z_{0}\right|<1,
\end{aligned}
$$

which proves (3.1) for $j=3$. To establish (3.2), we apply Lemma 1 to the function $p_{2}(z)$. According to (3.18) it follows now that

$$
\left|p_{2}^{\prime}(z)\right| \leqq \frac{(n+1) C(1,2)}{\left(1-|z|^{2}\right)^{3}},|z|<1,
$$

where by [7, proof of Lemma 4]

$$
C(1, k) \leqq 2 k+\left(\frac{1+2 k}{2 k}\right)^{k} \sqrt{1+2 k}, k=2,3, \cdots .
$$

Combining $\left(3.20^{\prime}\right)$ with $\left(3.22^{\prime \prime}\right)$, we conclude that

$$
\begin{aligned}
\left|p_{3}\left(z_{0}\right)\right| & \leqq \frac{A(3, n)+\frac{3}{2}(n+1) C(1,2)}{\left(1-\left|z_{0}\right|^{2}\right)^{3}} \\
& =\frac{B(3, n)}{\left(1-\left|z_{0}\right|^{2}\right)^{3}},\left|z_{0}\right|<1 .
\end{aligned}
$$

The general step in the induction is similar to the proof of the case $j=3$. We assume now that (3.1) and (3.2) were established for $j=2,3, \cdots, m, m \leqq n-1$. Since by the induction assumption the coefficients $p_{2}(z), \cdots, p_{m}(z)$ satisfy (3.2), if follows by Lemma 1 that

$$
\begin{aligned}
\left|p_{j}^{(m+1-j)}(z)\right| & \leqq \frac{B(j, n) C(m+1-j, j)}{\left(1-|z|^{2}\right)^{m+1}} \\
& \leqq \frac{M(m+1, n)}{\left(1-|z|^{2}\right)^{m+1}}, j=2, \cdots, m,|z|<1,
\end{aligned}
$$

where $M(m+1, n)$ is a constant depending only on $m$ and $n$. Note that for $z=0$ we may use the Cauchy inequality instead of Lemma 1 and thus obtain the better estimate

$\left(3.24^{\prime}\right)\left|p_{j}^{(m+1-j)}(0)\right| \leqq B(j, n) \operatorname{Min}_{0 \leqq r<1}\left\{r^{-m-1+j}\left(1-r^{2}\right)^{-j}\right\}, j=2, \cdots, m$.

Setting $t=m+1$ in (3.13), it follows from (3.11), the induction as- 
sumption and $\left(3.24^{\prime}\right)$ that

$$
\left|p_{m+1}(0)\right| \leqq B_{0}(m+1, n)
$$

and

$$
\left|I_{m+1}(0)\right|=\left|\sum_{s=2}^{m+1} a_{m+1, s} p_{s}^{(m+1-s)}(0)\right| \leqq A(m+1, n),
$$

where $B_{0}(m+1, n)$ and $A(m+1, n)$ are constants depending on $m$ and $n$ only. Since (3.25) holds with $I_{m+1}(0)$ replaced by $J_{m+1}(0)$, it follows from (3.15) and (3.17) that

$$
\left|I_{m+1}\left(z_{0}\right)\right|=\left|J_{m+1}(0)\right|\left|\zeta^{\prime}\left(z_{0}\right)\right|^{m+1} \leqq \frac{A(m+1, n)}{\left(1-\left|z_{0}\right|^{2}\right)^{m+1}},\left|z_{0}\right|<1 .
$$

Combining (3.25') with (3.24), we conclude that (3.2) holds for $j=$ $m+1 \leqq 1$. This completes the proof of the main statement of the theorem.

Starpness of Theorem 2 will be discussed in the following section by means of an example.

4. Example. Let $u(z)$ and $v(z)$ be linearly independent solutions of the second order differential equation

$$
w^{\prime \prime}(z)+s(z) w(z)=0 .
$$

If

$$
w_{i}(z)=m_{i} u(z)+n_{i} v(z), i=1,2, \cdots, n-1,
$$

where $m_{i}$ and $n_{i}, i=1, \cdots,(n-1)$, are arbitrary complex constants, then

$$
y(z)=\prod_{i=1}^{n-1} w_{i}(z)=\prod_{i=1}^{n-1}\left[m_{i} u(z)+n_{i} v(z)\right]
$$

is the general solution of a differential equation of order $n$. Note that $y(z)$ can also be represented as a polynomial of $u(z)$ and $v(z)$; namely

$$
y(z)=c_{1}[u(z)]^{n-1}+c_{2}[u(z)]^{n-2} v(z)+\cdots+c_{n}[v(z)]^{n-1},
$$

where $c_{1}, \cdots, c_{n}$ are arbitrary complex constant. We now apply a process given by Fano [3, p. 531-532] to obtain the explicit form of the differential equation satisfied by (4.2). Let

$$
F_{0}(z)=y(z), F_{1}(z)=y^{\prime}(z)
$$

and set 


$$
F_{k+1}(z)=F_{k}^{\prime}(z)+k(n-k) s(z) F_{k-1}(z), k, 1,2, \cdots, .
$$

It is easily verified by induction that if $y(z)$ is given by (4.2), then

$$
\begin{aligned}
& F_{0}=w_{1} w_{2} \cdots w_{n-1}, \\
& F_{1}=\sum_{i} w_{1} \cdots w_{i-1} w_{i}^{\prime} w_{i+1} \cdots w_{n-1}, \\
& F_{2}=2 ! \sum_{i<j} w_{1} \cdots w_{i-1} w_{i}^{\prime} w_{i+1} \cdots w_{j-1} w_{j}^{\prime} w_{j+1} \cdots w_{n-1},
\end{aligned}
$$

and

$$
F_{k}=k ! \sum w_{1}^{\left(\varepsilon_{1}\right)} \cdots w_{n-1}^{\left(\varepsilon_{n}-1\right)}, 0 \leqq k \leqq n-1 .
$$

Here the summation is over all possible sequences $\varepsilon_{1}, \cdots, \varepsilon_{n-1}, \varepsilon_{i}=0,1$, such that $\sum_{i=1}^{n-1} \varepsilon_{i}=k$; and $w_{i}^{(0)}=w_{i}, w_{i}^{(1)}=w_{i}^{\prime}$. Thus,

$$
F_{n-1}=(n-1) ! w_{1}^{\prime} w_{2}^{\prime} \cdots w_{n-1}^{\prime}
$$

and by (4.5) it follows now that

$$
F_{n}=F_{n-1}^{\prime}+(n-1) s F_{n-2} \equiv 0 \text {. }
$$

On the other hand, according to (4.4) and (4.5)

$$
\begin{gathered}
F_{0}=y, F_{1}=y^{\prime}, F_{2}=F_{1}^{\prime}+(n-1) s F_{0}=y^{\prime \prime}+(n-1) s y, \\
F_{3}=F_{2}^{\prime}+2(n-2) s F_{1}=y^{\prime \prime \prime}+(3 n-5) s y^{\prime}+(n-1) s y, \\
F_{4}=y^{(4)}+(6 n-14) s y^{\prime \prime}+(4 n-6) s^{\prime} y^{\prime} \\
\quad+\left[(n-1) s^{\prime \prime}+3(n-3)(n-1) s^{2}\right] y,
\end{gathered}
$$

and

$$
\begin{aligned}
F_{n}=y^{(n)} & +\left(\begin{array}{c}
n+1 \\
3
\end{array}\right) s y^{(n-2)}+2\left(\begin{array}{c}
n+1 \\
4
\end{array}\right) s^{\prime} y^{(n-3)} \\
& +3\left(\begin{array}{c}
n+1 \\
5
\end{array}\right)\left[s^{\prime \prime}+\frac{5 n+7}{9} s^{2}\right] y^{(n-4)} \\
& +4\left(\begin{array}{c}
n+1 \\
6
\end{array}\right)\left[s^{\prime \prime \prime}+\frac{5 n+7}{2} s s^{\prime}\right] y^{(n-5)}+\cdots
\end{aligned}
$$

(cf. [2, p. 236], [3, p. 531]). Combining (4.6) and (4.7) we conclude that (4.2) is the general solution of the differential equation

$$
\begin{aligned}
y^{(n)} & +\left(\begin{array}{c}
n \\
2
\end{array}\right) \frac{(n+1)}{3} s y^{(n-2)}+\left(\begin{array}{c}
n \\
3
\end{array}\right) \frac{(n+1)}{2} s^{\prime} y^{(n-3)}+\cdots \\
& +\left(\begin{array}{c}
n \\
j
\end{array}\right) p_{j} y^{n-j}+\cdots+p_{n} y=0 .
\end{aligned}
$$

Here $p_{j}(z), j=2, \cdots, n$, is a polynomial of the arguments $s^{(t)}(z), t=$ $0, \cdots, j-2$, with positive coefficients. Moreover, by $(4.5), p_{j}(z)$ is a 
homogeneous polynomial of weight $j$ provided $\left[s^{(t)}(z)\right]^{m}$ is of weight $m(t+2)$.

We assert now: (4.8) is disconjugate in the domain $D$, if and only if (4.1) is disconjugate in D. Indeed, according to (4.2), a solution $y(z)$ of (4.8) vanishes $n$ times in $D$, if and only if one of the solutions $w_{i}(z), 1 \leqq i \leqq n-1$, of equation (4.1) vanishes at least twice in $D$. Note that if (4.8) has a nontrivial solution which vanishes $n$ times in $D$, then there exists also a solution which has two zeros each of order $(n-1)$ in $D$. Furthermore, $(4.8)$ is nonoscillatory in $D$, (i.e., every solution of (4.8) has a finite number of zeros in $D$ ) if and only if (4.1) is nonoscillatory in $D$.

Let

$$
s(z)=\frac{a}{\left(1-z^{2}\right)^{2}},
$$

then according to a result of Hille [4], equation (4.1) is disconjugate in $|z|<1$, if and only if $a \in C$, where $C$ denotes the interior and the boundary of the cardioid given by $a=-2 e^{i \phi}-e^{2 i \phi}$. This cardioid goes through the points $a=+1$ and $a=-3$, contains $|a| \leqq 1$ and is contained in $|a| \leqq 3$. By the assertion made above, it follows now that (4.8) is disconjugate in $|z|<1$, if $s(z)$ is given by (4.9) and $a \in C$. Substitution of (4.9) in (4.8) leads us to a differential equation of the form (1.1), whose first coefficients are given by

$$
\begin{aligned}
p_{2}(z) & =\frac{(n+1) s(z)}{3}=\frac{(n+1) a}{3\left(1-z^{2}\right)^{2}}, \\
p_{3}(z) & =\frac{(n+1) s^{\prime}(z)}{2}=\frac{2(n+1) a z}{\left(1-z^{2}\right)^{3}} \\
p_{4}(z) & =\frac{3(n+1)}{5}\left[s^{\prime \prime}(z)+\frac{5 n+7}{9} s^{2}(z)\right] \\
& =\frac{3(n+1) a}{5\left(1-z^{2}\right)^{4}}\left[4+20 z^{2}+\frac{(5 n+7)}{9} a\right] .
\end{aligned}
$$

Setting $a=-3$ and $z=x, 0 \leqq x<1$, (4.10) yields

$$
\left|p_{2}(x)\right|=\frac{n+1}{\left(1-x^{2}\right)^{2}},\left|p_{3}(x)\right|=\frac{6(n+1) x}{\left(1-x^{2}\right)^{3}},
$$

which shows that (3.3) is sharp and the constants $A(2, n)=B(2, n)=$ $(n+1)$ are the best possible. For $3 \leqq j \leqq n,(4.8)$ and (4.9) show that (3.1) and (3.2) are of the correct order. Indeed, if $s(z)$ is given by (4.9), then

$$
\lim _{z \rightarrow 1} s^{(t)}(z)\left(1-z^{2}\right)^{t+2}=\lim _{z \rightarrow 1}(2 z)^{t}(t+1) ! a=2^{t}(t+1) ! a, t=0,1, \cdots .
$$


Since the coefficient $p_{j}(z)$ in (4.8) is a polynomial of the arguments $s(z), \cdots, s^{(j-2)}(z)$ with positive coefficients $\left(p_{j}(z)\right.$ is homogeneous of weight $j$, provided $\left[s^{t}(t)\right]^{m}$ is of weight $\left.m(t+2)\right)$, it follows that

$$
\lim _{z \rightarrow 1} p_{j}(z)\left(1-z^{2}\right)^{j}=P_{j}(a)
$$

where $P_{j}(a)$ is a polynomial in $a$ with positive coefficients. Clearly, $\left|P_{j}(a)\right|>0$ for almost every $a \in C$, where $C$ denotes the interior and the boundary of the cardioid. Hence, we conclude that there exist differential equations of the form (1.1), which are disconjugate in $|z|<1$ and such that

$$
\lim _{z \rightarrow 1}\left|p_{j}(z)\right|\left(1-|z|^{2}\right)^{j}>0 \text {. }
$$

Moreover, for a fixed $n, \operatorname{Max}\left|P_{j}(a)\right|$, where $a \in C$, yields a lower bound for the constant $B(j, n)$. For example, by (4.10)

$$
p_{3}(a)=\lim _{z \rightarrow 1} P_{3}(z)\left(1-z^{2}\right)^{3}=2(n+1) a .
$$

Therefore,

$$
\operatorname{Max}_{a \in C}\left|P_{3}(\alpha)\right|=\left|P_{3}(-3)\right|=6(n+1) .
$$

Hence, $B(3, n) \geqq 6(n+1)$. Comparing with the results obtained in the proof of Theorem 2, we have according to $\left(3.21^{\prime}\right)$

$$
B(3, n)=A(3, n)+\frac{3}{2}(n+1) C(1,2) .
$$

It is easily verified that for equation (4.8) the invariant $I_{3}(z)$ vanishes identically. (Actually, as will be shown later, equation (4.8) is selfadjoint and therefore, according to Corollary 3 of Theorem 1, all its invariants of odd weight vanish identically.) Setting in $(4.11) A(3, n)=$ 0 (because $\left.I_{3}(z) \equiv 0\right)$ and $C(1,2) \leqq 7.5$ (see $(3.23)$ ), it follows that for self-adjoint equations

$$
6(n+1) \leqq B(3, n) \leqq 11.25(n+1) .
$$

We assert now that equation (4.8) is self-adjoint. To verify this assertion we note that according to (4.3)

$$
\eta=\frac{y(z)}{[v(z)]^{n-1}}=\sum_{k=1}^{n-1} c_{k}\left[\frac{u(z)}{v(z)}\right]^{k}=\sum_{k=1}^{n-1} c_{k}[t(z)]^{h}, t(z)=\frac{u(z)}{v(z)} .
$$

Hence, $\eta$ is a polynomial of order $(n-1)$ in $t(z)$ and therefore satisfies the differential equation

$$
\frac{d^{n} \eta}{d t^{n}}=0
$$


In order to obtain from (4.12) and (4.13) the differential equation satisfied by $y(z)$, we proceed as follows. (cf. [10, p. 46-47], [2, p. 235237].) Without loss of generality we may assume that the Wronskian $u^{\prime}(z) v(z)-u(z) v^{\prime}(z)$ of equation (4.1) is identically equal to 1 . Hence,

$$
\frac{d t}{d z}=\frac{u^{\prime}(z) v(z)-u(z) v^{\prime}(z)}{v^{2}(z)}=\frac{1}{v^{2}(z)},
$$

and therefore

$$
\frac{d}{d t}=v^{2}(z) \frac{d}{d z}
$$

Combining (4.12), (4.13) and (4.15), it follows that $y(z)$ satisfies the $n$-th order differential equation

$$
v^{2}(z) \frac{d}{d z} \cdots v^{2}(z) \frac{d}{d z} \cdot v^{2}(z) \frac{d}{d z} \cdot \frac{y(z)}{[v(z)]^{n-1}}=0 .
$$

In order to normalize (4.16) so that the coefficient of $y^{(n)}$ will be equal to 1 , we multiply by $[v(z)]^{-n-1}$ and obtain

$$
\frac{1}{[v(z)]^{n-1}} \frac{d}{d z} \cdots v^{2}(z) \frac{d}{d z} \cdot v^{2}(z) \frac{d}{d z} \cdot \frac{y(z)}{[v(z)]^{n-1}}=0 .
$$

Hence, equation (4.8) can be expressed in terms of a solution $v(z)$ of (4.1) in the form (4.16'). The symmetric form of equation (4.16') implies now (see [5, p. 126]) that equation (4.8) is self-adjoint whether $n$ is even or odd.

We conclude our discussion of equation (4.8) with the following observation. If equation (4.8) is disfocal in $|z|<1$, then it is also disconjugate there. Indeed, assume that equation (4.8) is disfocal in $|z|<1$, (i.e., no nontrivial solution of (4.8) satisfies

$$
y\left(z_{1}\right)=y^{\prime}\left(z_{2}\right)=\cdots=y^{(n-1)}\left(z_{n}\right)=0,
$$

where $\left|z_{i}\right|<1, i=1,2, \cdots, n$, ) then according to [8, Th. 7]

$$
|s(z)| \leqq \frac{\left[\left(\begin{array}{c}
n+1 \\
3
\end{array}\right)\right]^{-1}}{\left(1-|z|^{2}\right)^{2}},|z|<1,
$$

which is sufficient [9, Th. 1] to imply the disconjugacy of equation (4.1) in $|z|<1$. Consequently, equation (4.8) is also disconjugate in $|z|<1$. (cf. [8, Th. 8].)

5. $m-m$ disconjugacy of self-adjoint differential equations. Considering the differential equation of even order 


$$
y^{(2 m)}(z)+p(z) y(z)=0,
$$

Kim has recently established the following theorem [6, Th. 2.1].

Let $p(z)$ be regular in $|z|<1$. If

$$
|p(z)| \leqq \frac{K(2 m)}{\left(1-|z|^{2}\right)^{2 m}},|z|<1,
$$

where

$$
K(2 m)=\prod_{i=0}^{m-1}(1+2 i)^{2}, m=1,2, \cdots,
$$

then the differential equation (5.1) is $m-m$ disconjugate in $|z|<1$; i.e., no (nontrivial) solution of (5.1) has two zeros of order $m$ in $|z|<1$. The constants (5.3) are the best possible. (Kim calls this property disconjugacy in the sense of Reid).

We generalize now Kim's result to self-adjoint differential equations of the form

$$
\begin{aligned}
y^{(2 m)}(z) & +\left[r_{2}(z) y^{m-1}(z)\right]^{(m-1)}+\cdots \\
& +\left[r_{2 k}(z) y^{(m-k)}(z)\right]^{(m-k)}+\cdots+r_{2 m}(z) y(z)=0 .
\end{aligned}
$$

THEOREM 3. Let $r_{2 k}(z), k=1,2, \cdots, m$, be regular in $|z|<1$. There exists positive constants $R(2 k, 2 m), k=1, \cdots, m$, depending only on $k$ and $m$, such that if

$$
\left|r_{2 k}(z)\right| \leqq \frac{R(2 k, 2 m)}{\left(1-|z|^{2}\right)^{2 k}},|z|<1, k=1, \cdots, m,
$$

then equation (5.4) is $m-m$ disconjugate in $|z|<1$.

As in [6], we require the following integral inequality.

LEMMA 2. Let $U(x)$ be a real function with s continuous derivatives in the interval $[-\rho, \rho]$. If $U(x)$ has two zeros of order $s$ at $\pm \rho$, then

$$
\int_{-\rho}^{\rho}\left[U^{(s)}(x)\right]^{2} d x>K(2 s) \rho^{2 s} \int_{-\rho}^{\rho} \frac{[U(x)]^{2}}{\left(\rho^{2}-x^{2}\right)^{2 s}}, s=1,2, \cdots,
$$

where $K(2 s)$ are given by (5.3).

Inequality (5.6) was established by Nehari [9] for $s=1$ and by Beesack [1] for $s=2$. Kim proved (5.6) for any natural number $s$ [6, Th. 3.3].

Proof of Theorem 3. We first prove that if (5.5) holds and 


$$
\sum_{k=1}^{m} \frac{R(2 k, 2 m)}{K(2 k)} \leqq 1,
$$

then no solution of (5.4) has two zeros of order $m$ at the symmetric points $\pm \rho, 0<|\rho|<1$. Suppose to the contrary, that there exists a solution $y(z)$ of (5.4) which vanishes $m$ times at $\pm \rho$. Without loss of generality we may assume that $\rho$ is real. Multiply now (5.4) by $\bar{y}(z)$ and integrate along the real axes from $-\rho$ to $\rho$. Integration by parts leads us to

$$
\int_{-\rho}^{\rho}\left|y^{(m)}(x)\right|^{2} d x=\sum_{k=1}^{m}(-1)^{m-k-1} \int_{-\rho}^{\rho} r_{2 k}(x)\left|y^{(m-k)}(x)\right|^{2} d x,
$$

since all the integrated parts vanish. Writing now $y(x)=u(x)+i v(x)$, we have $|y|^{2}=u^{2}+v^{2}$ and $\left|y^{(s)}\right|^{2}=\left(u^{(s)}\right)^{2}+\left(v^{(s)}\right)^{2}$. Thus, we obtain from $(5.8)$

$$
\int_{-\rho}^{\rho}\left[\left(u^{(m)}\right)^{2}+\left(v^{(m)}\right)^{2}\right] d x \leqq \sum_{k=1}^{m} \int_{-\rho}^{\rho}\left|r_{2 k}\right|\left[\left(u^{(m-k)}\right)^{2}+\left(v^{(m-k)}\right)^{2}\right] d x .
$$

By (5.5), it follows from $\left(5.8^{\prime}\right)$ that

$$
\begin{aligned}
& \int_{-\rho}^{\rho}\left[\left(u^{(m)}\right)^{2}+\left(v^{(m)}\right)^{2}\right] d x \\
& \leqq \sum_{k=1}^{m} \int_{-\rho}^{\rho} \frac{R(2 k, 2 m)}{\left(1-x^{2}\right)^{2 k}}\left[\left(u^{(m-k)}\right)^{2}+\left(v^{(m-k)}\right)^{2}\right] d x \\
& \leqq \sum_{k=1}^{m} \rho^{2 k} \int_{-\rho}^{\rho} \frac{R(2 k, 2 m)}{\left(\rho^{2}-x^{2}\right)^{2 k}}\left[\left(u^{(m-k)}\right)^{2}+\left(v^{(m-k)}\right)^{2}\right] d x .
\end{aligned}
$$

Since $y(x)=u(x)+i v(x)$ is supposed to have zeros of order $m$ at $\pm \rho$, the same is true for $u(x)$ and $v(x)$ separately. Applying Lemma 2 to the real functions $u^{(m-k)}(x)$ and $v^{(m-k)}(x)$ we obtain

$$
\rho^{2 k} \int_{-\rho}^{\rho} \frac{\left(u^{(m-k)}\right)^{2}+\left(v^{(m-k)}\right)^{2}}{\left(\rho^{2}-x^{2}\right)^{2 k}} d x<\frac{1}{K(2 k)} \int_{-\rho}^{\rho}\left[\left(u^{(m)}\right)^{2}+\left(v^{(m)}\right)^{2}\right] d x
$$

Hence, it follows from (5.9) and (5.10) that

$$
\int_{-\rho}^{\rho}\left[\left(u^{(m)}\right)^{2}+\left(v^{(m)}\right)^{2}\right] d x<\sum_{k=1}^{m} \frac{R(2 k, 2 m)}{K(2 k)} \int_{-\rho}^{\rho}\left[\left(u^{(m)}\right)^{2}+\left(v^{(m)}\right)^{2}\right] d x,
$$

which by (5.7) yields the desired contradiction.

We turn now to the general case and we assume that (5.5) is satisfied. We shall prove that if the positive constants $R(2 k, 2 m), k=$ $1, \cdots, m$, are taken small enough, then equation (5.4) is $m-m$ disconjugate in $|z|<1$. Suppose to the contrary, that there exists a solution $y(z)$ of equation (5.4) with two zeros of order $m$ at $z_{1}$ and $z_{2}$, where $z_{1}$ and $z_{2}$ are two (not necessarily symmetric) points in the unit 
disk. We apply now a transformation of the type (2.17). It is well known [9] that by a suitable choice of the parameters $z_{0}$ and $\theta$ in (2.17), it is possible to map $|z|<1$ onto $|\zeta|<1$ in such a way that $z_{1}$ and $z_{2}$ are mapped on two symmetric points of the real axes $\pm \rho$, $0<\rho<1$. By Corollary 4 of Theorem 1 the self-adjoint differential equation (5.4) is transformed now into the self-adjoint differential equation

$$
\begin{aligned}
w^{(2 m)}(\zeta) & +\left[s_{2}(\zeta) w^{(m-1)}(\zeta)\right]^{(m-1)}+\cdots \\
& +\left[s_{2 k}(\zeta) w^{(m-k)}(\zeta)\right]^{(m-k)}+\cdots+s_{2 m}(\zeta) w(\zeta)=0 .
\end{aligned}
$$

It follows now from our hypothesis that equation (5.11) has a solution which vanishes $m$ times at $\pm \rho$. Using Theorem 1 and Lemma 1 we shall show that (5.5) implies that

$$
\left|s_{2 k}(\zeta)\right| \leqq \frac{S(2 k, 2 m)}{\left(1-|z|^{2}\right)^{2 k}},|\zeta|<1, k=1, \cdots, m,
$$

where $S(2 k, 2 m), k=1, \cdots, m$, are constants which depend on $k$ and $m$ and on the constants $R(2 t, 2 m), t=1, \cdots, k$, but not on the choice of the parameters $z_{0}$ and $\theta$ in (2.17). Moreover $S(2 k, 2 m)$ is a linear homogeneous combination of the constants $R(2 t, 2 m), t=1, \cdots, k$. Thus, if $R(2 k, 2 m), k=1, \cdots, m$, are small enough, it is possible to guarantee that $S(2 k, 2 m)$ will satisfy

$$
\sum_{k=1}^{m} \frac{S(2 k, 2 m)}{K(2 k)} \leqq 1 .
$$

However, if the coefficients $s_{2 k}(\zeta)$ satisfy (5.12) and $\left(5.7^{\prime}\right)$ it follows from the first part of our proof that no (nontrivial) solution of equation (5.11) has two zeros of order $m$ at $\pm \rho, 0<|\rho|<1$; and this contradicts our hypothesis. Consequently, no solution of equation (5.4) has two zeros of order $m$ at $z_{1}$ at $z_{2}$, where $\left|z_{1}\right|,\left|z_{2}\right|<1$.

We now give the details. Since equation (5.4) is self-adjoint, it follows from Corollary 3 of Theorem 1 that the invariants of odd weight vanish identically; i.e.,

$$
I_{3}(z) \equiv I_{5}(z) \equiv \cdots \equiv I_{2 m-1}(z) \equiv 0 .
$$

By comparing the forms of equations (5.4) and (1.1) it follows from (2.9) that

$$
I_{2 k}(z)=\sum_{t=1}^{k} \alpha_{2 k, 2 t} r_{2 t}^{(2 k-2 t)}(z), k=1, \cdots, m,
$$

where $\left[\alpha_{2 k, 2 t}\right]_{1}^{m}$, is a triangular constant matrix whose elements are determined by the constants (2.11) and by the order $2 \mathrm{~m}$. In particular 
$\alpha_{2 k, 2 k}=\left[\left(\begin{array}{c}2 m \\ 2 k\end{array}\right)\right]^{-1}, k=1, \cdots, m$. Moreover, successive elimination of $r_{2}(z), \cdots, r_{2 m}(z)$ from (5.14) yields

$$
r_{2 k}(z)=\sum_{t=1}^{k} \beta_{2 k, 2 t} I_{2 t}^{(2 k-2 t)}(z), k=1, \cdots, m,
$$

where the triangular matrix $\left[\beta_{2 k, 2 t}\right]_{1}^{m}$ is the inverse of the triangular matrix $\left[\alpha_{2 k, 2 t}\right]_{1}^{m}$. (see the remark following the proof of Theorem 1.)

Since we assume that (5.5) is satisfied, it follows by Lemma 1 that

$$
\begin{gathered}
\left|r_{2 t}^{(2 k-2 t)}(z)\right| \leqq \frac{C(2 k-2 t, 2 t) R(2 t, 2 m)}{\left(1-|z|^{2}\right)^{2 k}}, \\
|z|<1, k=1, \cdots, m .
\end{gathered}
$$

Combining (5.14) and (5.16) we conclude that

$$
\left|I_{2 k}(z)\right| \leqq \frac{E(2 k, 2 m)}{\left(1-|z|^{2}\right)^{2 k}},|z|<1, k=1, \cdots, m .
$$

where

(5.18) $E(2 k, 2 m)=\sum_{t=1}^{k} \alpha_{2 k, 2 t} C(2 k-2 t, 2 t) R(2 t, 2 m), k=1, \cdots, m$.

Clearly, the constants $E(2 k, 2 m), k=1, \cdots, m$ can be made as small as we wish by taking $R(2 t, 2 m), t=1, \cdots, m$ small enough.

Denote by $J_{j}(\zeta), j=2, \cdots, 2 m$ the invariants of equation (5.11), then according to Theorem 1

$$
I_{j}(z)=J_{j}[\zeta(z)]\left[\zeta^{\prime}(z)\right]^{j}, j=2, \cdots, 2 m,
$$

where $\zeta(z)$ is the transformation (of the type (2.17)) which maps $|z|<1$ onto $|\zeta|<1$ and $z_{1}$ and $z_{2}$ to $\pm \rho$. By (5.13), (5.17) and (3.17), it follows from (2.10) that

$$
J_{3}(\zeta) \equiv J_{5}(\zeta) \equiv \cdots J_{2 m-1}(\zeta) \equiv 0
$$

and

$$
\left|J_{2 k}(\zeta)\right| \leqq \frac{E(2 k, 2 m)}{\left(1-|\zeta|^{2}\right)^{2 k}},|\zeta|<1, k=1, \cdots, m .
$$

The relations between the coefficients $s_{2 k}(\zeta), k=1, \cdots, m$ of equation (5.11) and the invariants $J_{2 k}(\zeta), k=1, \cdots, m$, are given by

$$
J_{2 k}(\zeta)=\sum_{t=1}^{k} \alpha_{2 k, 2 t} s_{2 t}^{(2 k-2 t)}(\zeta), k=1, \cdots, m
$$

or by the equivalent relations 


$$
s_{2 k}(\zeta)=\sum_{t=1}^{k} \beta_{2 k, 2 t} I_{2 t}^{(2 k-2 t)}(\zeta), k=1, \cdots, m .
$$

Applying now Lemma 1 to $J_{2 k}(\zeta)$, it follows from (5.17') that

$$
\begin{gathered}
\left|J_{2 t}^{(2 k-2 t)}(\zeta)\right| \leqq \frac{C(2 k-2 t, 2 t) E(2 t, 2 m)}{\left(1-|\zeta|^{2}\right)^{2 k}}, \\
|\zeta|<1, t=1, \cdots, k .
\end{gathered}
$$

Substituting (5.19) in $\left(5.15^{\prime}\right)$ we arrive at (5.12) and the constants $S(2 k, 2 m)$ are given by

(5.20) $S(2 k, 2 m)=\sum_{t=1}^{k} \beta_{2 k, 2 t} C(2 k-2 t, 2 t) E(2 t, 2 m), k=1, \cdots, m$.

Combining (5.18) and (5.20) we conclude that $S(2 k, 2 m)$ is a linear homogeneous function of $R(2 i, 2 m), i=1, \cdots, k$. Therefore, the constants $S(2 k, 2 m), k=1, \cdots, m$, will satisfy $\left(5.17^{\prime}\right)$ provided $R(2 k, 2 m)$, $k=1, \cdots, m$, are small enough. This completes the proof of Theorem 3 .

For the fourth order self-adjoint equation

$$
y^{(4)}(z)+\left[r_{2}(z) y^{\prime}(z)\right]^{\prime}+r_{4}(z) y(z)=0 .
$$

Theorem 3 yields the following results. Let $r_{2}(z)$ and $r_{4}(z)$ satisfy (5.5).

(i) If

$$
R(2,4)+\frac{R(4,4)}{9} \leqq 1
$$

then no solution of (5.21) has double zeros at two symmetric points $\pm \rho, 0<|\rho|<1$.

(ii) If

$$
R(2,4)+\frac{R(4,4)+\frac{3}{5} C(2,2) R(2,4)}{9} \leqq 1
$$

then no solution of (5.21) has double zeros at any two points of the unit disk; i.e., (5.21) is $2-2$ disconjugate in $|z|<1$. Since $C(2,2) \leqq$ $C(1,2) C(1,3)$, it follows from $(3.23)$ that $C(2,2) \leqq 7.5 \times 10.2$ and $(5.22)$ takes the form

$$
6.1 R(2,4)+\frac{R(4,4)}{9} \leqq 1
$$

Theorem 3 can also be stated in terms of the invariants in the following way.

THEOREM 3 . Let $I_{j}(z), j=2, \cdots, 2 m$ be regular functions in 
$|z|<1$, such that (5.13) and (5.17) are satisfied. Let $S(2 k, 2 m), k=$ $1, \cdots, m$, be defined by (5.20). If the positive constants $E(2 k, 2 m)$, $k=1, \cdots, m$, are small enough to guarantee that $\left(5.7^{\prime}\right)$ is satisfied, then equation (1.1) is $m-m$ disconjugate in $|z|<1$.

For fourth order equations Theorem $3^{\prime}$ yields: Let $I_{3}(z) \equiv 0$ and let $I_{2}(z)$ and $I_{4}(z)$ satisfy (5.17). If

$$
6\left(1+\frac{C(2,2)}{30}\right) E(2,4)+\frac{E(4,4)}{9} \leqq 1,
$$

then the differential equation is $2-2$ disconjugate in $|z|<1$.

We conclude with the following remark. As has been shown in the end of $\S 4$, equation (4.8) is self-adjoint. Moreover, if $n=2 m$, then equation (4.8) is $m-m$ disconjugate in $|z|<1$, if and only if it is disconjugate there. Setting now $s(z)=a\left(1-z^{2}\right)^{-2-\delta}, \delta>0$, in (4.8), it follows from Theorem 2 that for any choice of the complex constant $a$ and the positive constant $\delta$, equation (4.8) is not disconjugate and therefore also not $m-m$ disconjugate in $|z|<1$. Hence, (5.5) and (5.17) are of the right order of growth. Indeed, no condition of the type

$$
\begin{gathered}
\left|I_{2 k}(z)\right| \leqq \frac{E(2 k, 2 m)}{\left(1-|z|^{2}\right)^{2 k+\varepsilon}},|z|<1, \varepsilon>0, \\
E(2 k, 2 m)>0, k=1, \cdots, m,
\end{gathered}
$$

can possibly imply $m-m$ disconjugacy of the self-adjoint differential equation (5.4) in $|z|<1$, however small the positive constants $E(2 k, 2 m)$ and $\varepsilon$ may be.

I am grateful to Professors Z. Nehari and B. Schwarz for their valuable advice.

\section{REFERENCES}

1. P. R. Beesack, Integral inequalities of the Wirtinger type, Duke Math. J. 25 (1958), 477-498.

2. F. Brioschi, Les invariants des équations différentieles linéaires, Acta Math. 14 (1890), 223-248.

3. Gino Fano, Ueber lineare homogene Differentialgleichungen mit algebraischen Relationen zwischen den Fundamentallosungen, Math. Ann. 53 (1900), 493-590.

4. E. Hille, Remarks on a paper by Z. Nehari, Bull. Amer. Math. Soc. 55 (1949), $552-553$.

5. E. L. Ince, Ordinary differential equations, Dover Publications Inc., 1956.

6. W. J. Kim, On the zeros of solutions of $y^{(n)}+p y=0$, J. Math. Anal. Appl. 25 (1969), 189-208.

7. M. Lavie, The Schwarzian derivative and disconjugacy of $n$-th order linear differential equations, Canad. J. Math. 21 (1969), 235-249. 
8.

Some function-theoretic aspects of disconjugacy of linear differential systems, Trans. Amer. Math. Soc. (1969) to appear.

9. Z. Nehari, The Schwarzian derivative and schlicht functions, Bull. Amer. Math. Soc. 55 (1949), 545-551.

10. E. J. Wilczynski, Projective differential geometry of curves and ruled surfaces, Chelsea Publishing Company, New York.

Received April 7, 1969. This research was sponsored by the Army Research Office Grant No. DA-ARO-D-31-124-G951 and Air Force Office of Scientific Research Grant No. AF-AFOSR-62-414.

CARNEGIE-MELLON UNIVERSITY

Pittsburgh, Pennsyluania

TEChNiON-ISRAEL INSTITUTE OF TEChNOLOGY

HAIFA, ISRAEL 



\section{PACIFIC JOURNAL OF MATHEMATICS}

\section{EDITORS}

\author{
H. SAMELSON \\ Stanford University \\ Stanford, California 94305 \\ RICHARD PIERCE \\ University of Washington \\ Seattle, Washington 98105
}

J. DugundJI

Department of Mathematics University of Southern California Los Angeles, California 90007

BASIL GORDON*

University of California

Los Angeles, California 90024

\section{ASSOCIATE EDITORS}
E. F. BECKENBACH
B. H. NEUMANN
F. WOLE
K. YOSHIDA

\section{SUPPORTING INSTITUTIONS}

\author{
UNIVERSITY OF BRITISH COLUMBIA \\ CALIFORNIA INSTITUTE OF TECHNOLOGY \\ UNIVERSITY OF CALIFORNIA \\ MONTANA STATE UNIVERSITY \\ UNIVERSITY OF NEVADA \\ NEW MEXICO STATE UNIVERSITY \\ OREGON STATE UNIVERSITY \\ UNIVERSITY OF OREGON \\ OSAKA UNIVERSITY \\ UNIVERSITY OF SOUTHERN CALIFORNIA
}

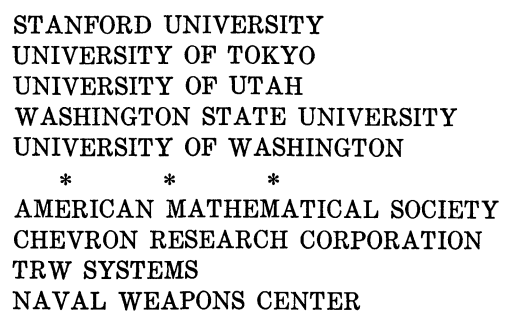

The Supporting Institutions listed above contribute to the cost of publication of this Journal, but they are not owners or publishers and have no responsibility for its content or policies.

Mathematical papers intended for publication in the Pacific Journal of Mathematics should be in typed form or offset-reproduced, (not dittoed), double spaced with large margins. Underline Greek letters in red, German in green, and script in blue. The first paragraph or two must be capable of being used separately as a synopsis of the entire paper. The editorial "we" must not be used in the synopsis, and items of the bibliography should not be cited there unless absolutely necessary, in which case they must be identified by author and Journal, rather than by item number. Manuscripts, in duplicate if possible, may be sent to any one of the four editors. Please classify according to the scheme of Math. Rev. 36, 1539-1546. All other communications to the editors should be addressed to the managing editor, Richard Arens, University of California, Los Angeles, California, 90024.

50 reprints are provided free for each article; additional copies may be obtained at cost in multiples of 50 .

The Pacific Journal of Mathematics is published monthly. Effective with Volume 16 the price per volume (3 numbers) is $\$ 8.00$; single issues, $\$ 3.00$. Special price for current issues to individual faculty members of supporting institutions and to individual members of the American Mathematical Society: $\$ 4.00$ per volume; single issues $\$ 1.50$. Back numbers are available.

Subscriptions, orders for back numbers, and changes of address should be sent to Pacific Journal of Mathematics, 103 Highland Boulevard, Berkeley, California, 94708.

PUBLISHED BY PACIFIC JOURNAL OF MATHEMATICS, A NON-PROFIT CORPORATION

Printed at Kokusai Bunken Insatsusha (International Academic Printing Co., Ltd.), 7-17, Fujimi 2-chome, Chiyoda-ku, Tokyo, Japan.

* Acting Managing Editor. 


\section{Pacific Journal of Mathematics}

\section{Vol. 32, No. $2 \quad$ February, 1970}

Harry P. Allen and Joseph Cooley Ferrar, Jordan algebras and exceptional subalgebras of the exceptional algebra $E_{6} \ldots \ldots \ldots \ldots \ldots \ldots \ldots 283$

David Wilmot Barnette and Branko Grünbaum, Preassigning the shape of a

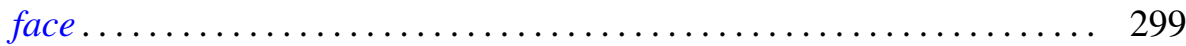

Robert Francis Craggs, Involutions of the 3-sphere which fix 2-spheres . . . . 307

David William Dean, Bor-Luh Lin and Ivan Singer, On k-shrinking and $k$-boundedly complete bases in Banach spaces ................ 323

Martin Engert, Finite dimensional translation invariant subspaces ....... 333

Kenneth Lewis Fields, On the global dimension of residue rings ......... 345

Howard Gorman, The Brandt condition and invertibility of modules ....... 351

Benjamin Rigler Halpern, A characterization of the circle and interval ..... 373

Albert Emerson Hurd, A uniqueness theorem for second order quasilinear

hyperbolic equations ............................... 415

James Frederick Hurley, Composition series in Chevalley algebras ...... 429

Meira Lavie, Disconjugacy of linear differential equations in the complex

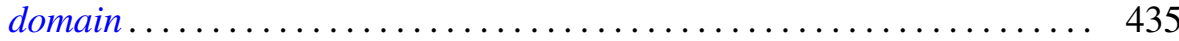

Jimmie Don Lawson, Lattices with no interval homomorphisms ......... 459

Roger McCann, A classification of center-foci ................. 467

Evelyn Rupard McMillan, On continuity conditions for functions . . . . . . . 479

Graciano de Oliveira, A conjecture and some problems on permanents .... 495

David L. Parrott and S. K. Wong, On the Higman-Sims simple group of order $44,352,000$.

Jerome L. Paul, Extending homeomorphisms ................. 517

Thomas Benny Rushing, Unknotting unions of cells .............. 521

Peter Russell, Forms of the affine line and its additive group.......... 527

Niel Shilkret, Non-Archimedean Gelfand theory ................. 541

Alfred Esperanza Tong, Diagonal submatrices of matrix maps.......... 551 